\title{
THE GLOBAL PROPERTIES OF PLANETARY NEBULAE IN THE GALACTIC BULGE
}

\author{
G. STASIŃSKA \\ DAEC, Observatoire de Paris-Meudon, France
}

\begin{abstract}
After a discussion of the methods used to derive the physical parameters of planetary nebulae and their central stars, the global properties of planetary nebulae in the Galactic bulge are reviewed, and compared to those of planetary nebulae in different contexts.
\end{abstract}

\section{Introduction}

Planetary nebulae (PN) are thought to be the evolution product of intermediate mass stars -0.8 to $8 \mathrm{M}_{\odot}$ - before they become white dwarfs (see recent reviews by Habing 1990 or Pottasch 1992). They are formed of matter ejected by these stars after the asymptotic giant branch stage and are gradually ionized as the core temperature grows.

It is natural to expect that the study of a population of PN should reveal some of the characteristics of the parent stars: ages, stellar masses and chemical composition, kinematics. Such was indeed the motivation of some of the former studies of Galactic bulge planetary nebulae (GBPN) like those of Webster $(1975,1976)$ or Isaacman (1983).

Further, the study of GBPN is a necessary step towards the understanding of the final evolutionary stages of intermediate mass stars. Indeed, most of the observational tests of existing theories require the knowledge of PN distances. Unfortunately, because PN are essentially transient objects, there is no quantity which remains constant during their evolution and could be used as a distance indicator. Together with the Magellanic Clouds, the Galactic bulge offers the possibility to study a population of $\mathrm{PN}$ at known distance, and this has been the motivation of many recent studies of GBPN (Gathier et al 1983, Pottasch and Acker 1989, Pottasch et al 1988, 1990, Pottasch 1990, Dopita et al 1990, Ratag 1991, Stasinska et al 1991a, b, Tylenda et al 1991, Pottasch and Zijlstra 1992). Unexpectedly, these studies have perpetuated some of the controversies regarding the interpretation of PN. 


\section{The GBPN sample}

\subsection{METHOD OF DISCOVERY}

The first systematic PN searches were made in the optical, either with objective prisms to identify characteristic emission lines (e.g. Minkowski 1948-1951, see references in Minkowski 1965), or on Palomar plates where are seen PN of large angular diameters and low surface brightness (Abell 1955). Deeper surveys of selected areas have been performed since then (see references in the Strasbourg-ESO catalogue of Galactic planetary nebulae, Acker et al 1992).

Interstellar extinction towards the bulge is large, hampering the detection of faint PN, except in some regions like the Baade Window which has been extensively studied by Kinman et al (1988).

Planetary nebulae can also be discovered by their radio continuum emission, but the risk of confusion with compact HII regions and especially extragalactic sources is important.

A more promising way is to select potential PN from IRAS sources on the basis of their infrared colours (Pottasch et al 1990) and then confirm their nature by further observations. The first results of a vast program of this kind are given in Pottasch et al (1988), Ratag et al (1990), Ratag and Pottasch (1991) for radio continuum measurements, and Manchado et al (1989) and Garcia-Lario et al (1990) for near-infrared photometry .

An optical spectrophotometric survey of all candidate GBPN published by 1988 has been conducted by Acker et al (1991), resulting in the confirmation of $335 \mathrm{PN}$ in the direction of the bulge. The criteria adopted to classify an object as a PN are presented in Acker et al (1987). While it is easy to distinguish an emission line galaxy from a PN, thanks to the redshift, it is more difficult to decide between a very young PN and a symbiotic star or a compact HII region. Any criteria presuppose a certain representation of the PN phenomenon. It is instructive to compare the lists of misclassified PN of Acker and Stenholm (1990) and Kohoutek (1992).

\subsection{SORTING OUT TRUE GBPN}

As noted by Minkowski (1965), the strong concentration of known Galactic PN towards the bulge indicates that most of the PN seen in that part of the sky actually belong to the bulge. The construction of separate histograms for PN with large ( $>20 ")$ and small $(<20 ")$ angular diameters led Gathier et al (1983) to estimate that about $80 \%$ of all the small PN within $10^{\circ}$ of the Galactic center are physically close to it. After rejecting those PN with radio flux $\mathrm{F}(6 \mathrm{~cm})$ above $100 \mathrm{mJy}$, among which a large proportion of foreground object is expected, the resulting sample should contain $90-95 \%$ of genuine GBPN (Pottasch and Acker 1989, Stasinska et 
al 1991). Further arguments, like for example a small distance as measured from the reddening(Gutiérrez-Moreno et al 1991, Ratag et al 1992) can be used to eliminate a few additional objects.

\section{A first glance at the GBPN sample}

\subsection{RADIAL VELOCITIES}

Using radial velocity measurements of about 300 galactic PN, Minkowski (1965) showed that in the Galactic bulge, many PN are on non-circular orbits, contrary to the majority of PN situated in the Galactic disk. Data on radial velocities are now available for 577 PN (Acker et al 1992), and confirm that most GBPN are of extreme Population II. According to Pottasch (1984), their central stars should thus have the same age as globular clusters, i.e. about 10-15 Gyrs. Since the stars had to take this long to evolve into $\mathrm{PN}$, their initial masses must have been about $0.8 \mathrm{M}_{\odot}$. In comparison, the PN velocity dispersion in the solar neighbourhood leads to an age of 3-5 Gyrs, corresponding to a progenitor masses of $1.4-2 \mathrm{M}_{\odot}$.

The average velocity of GBPN relative to their ambient medium is about $140 \mathrm{~km} / \mathrm{sec}($ Pottasch,1984) compared to $40 \mathrm{~km} / \mathrm{sec}$ for disk PN.

\subsection{EXCITATION CLASSES}

The "excitation class" of a nebula is an easily observed spectral property, as it relies only on the brightest lines. Therefore, it has become customary to use excitation classes to compare samples of PN in different environments. Several classification systems exist. Aller's (1956) favours line ratios independant of elemental abundances while Feast's (1968) minimizes reddening effects by considering lines in the same wavelength range.

Compared to PN located in the disk, GBPN have, on average, lower excitation classes (Acker et al 1991, Ratag 1991). They have also smaller excitation classes than PN in the LMC (Webster 1988). This is interpreted as due to a smaller mean effective temperature of the exciting stars in GBPN. However, this interpretation may not be correct: Schönberner and Tylenda (1990) have shown that the excitation class is not necessarily a good indicator of the effective temperature, because of the coupled evolution the star and the surrounding nebula.

\section{Determination of the physical properties of PN and their nuclei}

The methods mentionned below are classical and widely used in nebular studies (see e.g. Pottasch 1984). To enlighten the further discussion, we briefly recall the basics and limitations of the most important ones. 


\subsection{NEBULAR DENSITIES AND TEMPERATURES}

Nebular densities can be obtained from the ratios of collisionnally excited forbidden lines ([O II], [S II], [Cl III], [A IV]), which are sensitive to this parameter in the density range $10^{2}-10^{5} \mathrm{~cm}^{-3}$ approximately.

For PN at known distances, densities can also be derived from the angular diameter $\phi$ and the total reddening-free $H \beta$ (or radio) flux since $\mathrm{n}_{\mathrm{e}} \alpha \mathrm{F}(\mathrm{H} \beta)^{0.5} \phi^{-1.5} \mathrm{~d}^{-0.5}$. This method is believed to give a value more representative of the whole nebula than the line ratio method, which is very sensitive to density fluctuations .

The temperature of the ionized gas is given by the ratio of [O III] $4363 / 5007$ and sometimes [N II] 5755/6583 lines. In the case of densities above $10^{5} \mathrm{~cm}^{-3}$, these ratios can be misinterpreted, overestimating the temperatures. The electron temperatures of $\mathrm{PN}$ in the bulge range from $8000 \mathrm{~K}$ to $15000 \mathrm{~K}$. It is not excluded that some PN have lower temperatures which cannot be measured because the [O III] 4363 line is too faint. This could be the case of GBPN with abundances much higher than solar.

\subsection{MASSES OF THE NEBULAE}

In general, only the masses of the ionized portions of the PN can be readily obtained. Their derivation requires the knowledge of the distance and of the total $\mathrm{H} \beta$ (or radio) flux. The first method uses the density $\mathrm{n}_{\mathrm{e}}$ derived from forbidden line and the relation $\mathrm{M}_{\mathrm{i}} \alpha \mathrm{F}(\mathrm{H} \beta) \mathrm{n}_{\mathrm{e}} \mathrm{d}^{2}$. The other one uses the angular diameter $\phi$ and the relation: $\mathrm{M}_{\mathrm{i}} \alpha \mathrm{F}(\mathrm{H} \beta)^{0.5} \varepsilon^{0 .} 5_{\phi} 1.5 \mathrm{~d}^{2.5}$, where $\varepsilon$ is the filling factor, assumed of the order of 0.5. Both methods have their drawbacks. The first one because of the inhomogeneous structure of PN, the second one because of the large dependance of $\mathrm{M}_{\mathrm{i}}$ on the angular diameter which is uneasy to measure.

This last decade, observations of neutral and molecular gas in PN (see review by Huggins 1992) have started giving some information on the mass of the neutral gas may surround the optically shining PN (see Pottasch 1992), but there are yet no measurements for PN in the bulge.

\subsection{TEMPERATURES OF THE CENTRAL STARS}

Temperatures of PN nuclei can be estimated by different means.

One way is to use the nebular recombination lines to count the stellar ionizing photons, as proposed by Zanstra in 1931. Provided that the nebula is in balance between ionization and recombination (which is generally true) and absorbs all the stellar photons with energies above $13.6 \mathrm{eV}$ (which is not always true), the star effective temperature can be derived from the ratio of the nebular $\mathrm{H} \beta$ flux and the stellar flux in the $\mathrm{V}$ band. The nebular HeII 4686 line can be used instead $\mathrm{H} \beta$ to derive another estimate of the temperature. The chances for the nebula of being optically thick to $\mathrm{He}^{+}$ 
ionizing photons is much larger, but the star must be hotter than $510^{4} \mathrm{~K}$ to give a detectable nebular He II 4686 flux. A variant of this method, which does not require the measurement of the star magnitude, is to use the HeII $4686 / \mathrm{H} \beta$ ratio.

Another way to derive the star temperature is from the mean energy of the photons absorbed by the nebula, like in the energy balance method. This energy is given by the sum of all the collisionnally excited lines emitted by the nebula, normalized to $\mathrm{H} \beta$. This method has the advantage of being only weakly dependant on the optical thickness of the nebula in the Lyman continuum. It has been recently worked out by Preite-Martinez and Pottasch (1983), Preite-Martinez et al (1989) and Köppen and PreiteMartinez (1991). If the available spectra are restricted in wavelength (and this is generally the case, especially for GBPN) some corrections have to be introduced for the unobserved lines (mainly the UV carbon lines and the strongest infrared lines). An extreme version of the energy balance method - used recently by Méndez (1992) for GBPN - considers only the $[\mathrm{O}$ III]/H $\beta$ ratio, after calibration by an independent method.

Both these methods break down in the situation where dust competes with the gas in absorbing ionizing photons. This, however, appears to be the case only in very young PN (Lenzuni et al 1989).

The temperatures of PN nuclei can also be obtained as a result of photoionization modelling of the surrounding nebulae, such as was done by Ratag (1991) for about 100 GBPN. This method is not completely independent of the previous ones, since the first requirement of a model is to reproduce the HeII/H $\beta$ line (when observed), as well the intensities of the strongest lines, taking into account all the physical processes occuring in the nebula - which are schematized in the former two approaches.

The derived effective temperatures depend on the model atmospheres used for the exciting stars. Considerable progress has been made recently in the modelling of such atmospheres, with inclusion of non LTE effects in hydrogen and helium as well as in the metals, consideration of extended atmospheres and winds (see Kudritzki 1989 and 1992 for a review). It seems that, grossly, the effect of a stellar wind superimposed on a NLTE atmosphere is to restore the energy distribution towards a blackbody. Therefore, it is still sufficient for many purposes to assume that PN nuclei radiate as blackbodies (Dopita et al 1990, Méndez et al 1992).

Finally, these last years, it has become possible to derive the stellar parameters of the PN cores by direct spectroscopic non-LTE methods for about 20 objects (see Méndez et al 1992). These methods, however, are not devoid of problems (see Kudritzki 1992, Pottasch 1992). They are difficult to apply to GBPN, because they require good signal-to-noise spectra allowing the measurements of stellar line profiles. 
The discrepancies between the different derivations of star temperatures are often large (see e.g. Ratag 1991, or Pottasch 1992). They contain potential information on the properties of the PN and their exciting stars. Meanwhile, one should remain cautious about the significance of star temperature estimates.

\subsection{LUMINOSITIES OF THE CENTRAL STARS}

For PN at known distance, once the core temperatures are known, their luminosities are easily derived from their observed magnitudes by applying a bolometric correction. If the magnitudes are not available which is the case of about one third of known GBPN - core luminosities are sometimes estimated from the total nebular $\mathrm{H} \beta$ or radio flux (Pottasch and Acker 1989, Zijlstra and Pottasch 1989, Ratag 1991). If the nebula is density bounded or if dust absorbs part of the ionizing radiation, this procedure underestimates the stellar luminosity. Ratag (1991) has used the infra-red fluxes measured by IRAS to correct for dust absorption, but the problem of leakage of ionizing photons remains.

\subsection{MASSES OF THE CENTRAL STARS}

During the evolution of a PN, the luminosity of its nucleus varies by several orders of magnitude, and its temperature by a factor 10. A PN is intrinsically characterized by its core mass and its nebular mass and age.

The core mass can be derived by comparing measured stellar parameters with a grid of evolutionary models for post-AGB stars. By now, only two such grids are available: one for PN nuclei with a hydrogen burning shell (Schönberner 1979, 1983, Blöcker and Schönberner 1989), the other for both hydrogen and helium burning nuclei (Wood and Faulkner 1986). These grids cannot be combined, however, because the calculations were made under different assumptions (see a discussion by Schönberner 1989). A new grid is in preparation (Vassiliadis an Wood 1992), for different chemical compositions.

Several types of diagrams can be used to derive the PN core masses. All have their drawbacks (see a review by Tylenda 1992).

Errors in the luminosities or temperatures of PN nuclei affect the derived stellar masses. The application to density bounded PN of methods valid for ionization bounded ones leads to systematic effects. Tylenda et al (1991) have attempted to minimize them by comparing the position of the data points to "apparent theoretical evolutionary tracks" corresponding to models of nuclei surrounded by a standard expanding nebula and treated exactly like true objects in the derivation of the stellar parameters.

Finally, when the stars are still on their horizontal track in the H-R plane, a core-mass luminosity relation is expected. When considering a population of PN, the maximum luminosity corresponds to a maximum 
mass of the exciting stars. This is difficult to use in practice since, as noted by Pottasch (1992), different authors obtain different core-mass relations.

\subsection{CHEMICAL COMPOSITION OF THE NEBULAE}

Planetary nebulae are excellent sites for determining elemental abundances. Indeed, the process of formation of the emission lines used as abundance indicators is relatively well understood.

The simplest methods are empirical ones. The intensities of emission lines lead directly to ionic abundances (once the electron temperature is known). Elemental abundances are then obtained after correcting for unseen ionization stages. These corrections are adopted from simple consideration of ionization potentials (e.g. Köppen et al 1991), from grids of photoionization models (Walton et al 1992), or from modelling the ionization structure of individual PN (Aller and Keyes 1987, Ratag 1991).

Another method is to produce for each PN a photoionization model fitting all the lines. This painstaking method has seldom been applied. A simplified version was used by Dopita et al (1990) for 6 GBPN with isobaric nebular models and by Ratag (1991) for 120 GBPN with uniform density models. A perfect fit to all the observed lines was not looked for.

In GBPN, the abundances of $\mathrm{He}$ are considered to be accurate within $10-20 \%$, those of $\mathrm{O}$ within about $20-30 \%$, those of $\mathrm{N}$ and $\mathrm{Ne}$ within about $30-40 \%$. The $\mathrm{C}$ abundance has been determined only recently in a dozen of GBPN (Walton 1992), from IUE measurements. Abundances of S and A can also be also determined, the main problem lying in the ionization correction factors. Typical quoted errors are of 50-60\%. It is interesting to compare the abundances derived recently by 4 different groups of observers for M2-29, a halo PN located in the bulge.

\begin{tabular}{|c|c|c|c|c|c|c|c|}
\hline $\mathrm{He} / \mathrm{H}$ & $\begin{array}{l}\mathrm{C} / \mathrm{H} \\
\left(\times 10^{4}\right)\end{array}$ & $\begin{array}{l}\mathrm{N} / \mathrm{H} \\
\left(\mathrm{x} 10^{4}\right)\end{array}$ & $\begin{array}{l}\mathrm{O} / \mathrm{H} \\
\left(\times 10^{4}\right)\end{array}$ & $\begin{array}{l}\mathrm{Ne} / \mathrm{H} \\
\left(\mathrm{x} 10^{4}\right)\end{array}$ & $\begin{array}{l}\mathrm{S} / \mathrm{H} \\
\left(\mathrm{x} 10^{6}\right)\end{array}$ & $\begin{array}{l}\mathrm{Ar} / \mathrm{H} \\
\left(\mathrm{x} 16^{4}\right)\end{array}$ & \\
\hline 0.15 & & 0.13 & 0.6 & 0.2 & 2.5 & 2.0 & Dopita et al 1990 \\
\hline 0.13 & & 0.10 & 0.295 & 0.064 & 0.68 & 0.44 & Ratag 1991 \\
\hline 0.093 & 0.01 & 0.172 & 0.277 & 0.039 & & & Walton et al 1992 \\
\hline 0.091 & & 0.01: & 0.38 & 0.57 & 0.26 & & Köppen et al 1992 \\
\hline 0.116 & & $0.115 *$ & 3.88 & 1.01 & 1.25 & .90 & mean \\
\hline $25 \%$ & & $17 \% *$ & $38 \%$ & $86 \%$ & $87 \%$ & $107 \%$ & $\sigma$ \\
\hline
\end{tabular}

The scatter between the different abundance sets is larger than the uncertainty claimed by each group.

As shown by Peimbert (1967), temperature fluctuations in the nebula can lead one to underestimate the abundances of the heavy elements $(C, N$, $\mathrm{O}, \mathrm{Ne} . .$.$) relative to hydrogen. Up to now, there is no compelling$ 
observational or theoretical evidence for the existence of large temperature fluctuations but the problem is still open (see e.g. Ratag et al 1992).

\section{Some controversies in GBPN studies}

Even though the severest problem in PN studies - distances - is considerably reduced in the case of GBPN, their general properties are hard to establish because of difficulties in interpreting the observed data in terms of accurate physical parameters, and because objects of different evolutionary status may coexist. We describe below a few hot problems.

\subsection{MEASUREMENT UNCERTAINTIES}

A good example is that of the angular diameters, for which optical and VLA determinations may differ by a factor 2 or more (Stasinska et al 1991, Pottasch and Zijlstra 1992).

Another difficulty is the extinction correction, which must be applied to optical data. As shown by Stasinska and Tylenda (1992), the Balmer decrement method with the standard extinction law may overestimate the true fluxes by up to a factor $2-3$ if the reddening is large.

\subsection{INTERPRETATION OF OBSERVATIONAL DIAGRAMS}

A typical case is that of the mass-radius relation. Several authors (Pottasch and Acker 1989, Dopita et al 1990) have noted a clear correlation between the nebular ionized masses and diameters. The slope of 1.5 is exactly the one expected if the nebulae were gradually ionized by stars of constant ionizing flux, seeming to indicate that most GBPN are ionization bounded.

Zijlstra (1990) argued that observational selection must affect this diagram. Numerical simulations (Stasinska and Tylenda 1992) demonstrate that the observed diagram can be reproduced for a population of PN with only 0.3 dex dispersion in total masses, taking into account a $10 \%$ dispersion in the GBPN distances, combined with observational uncertainties and selection effects. In the simulation, more than $50 \%$ of PN turn out to be density bounded. For them, Zanstra temperatures and luminosities would be undersetimated.

\subsection{EVOLUTION OF THE CENTRAL STARS}

Recently, a number of number of authors (Pottasch and Acker 1989, Zijlstra and Pottasch 1989, Ratag et al 1992, Pottasch 1992) have reported the existence of young PN of low stellar luminosities, corresponding to core masses below $0.546 \mathrm{M}_{\odot}$. According to current evolutionary models of post AGB stars, such low mass nuclei would require more than $10^{5}$ years to become hot enough to ionize the ejected nebula. This is larger by about a factor five than the estimated expansion age of the PN. In fact, in the 
above papers, the luminosities were obtained under the assumption that the nebulae were ionization bounded. Tylenda et al (1991), as well as Méndez (1992), reanalyzing the data of Pottasch and Acker (1989), did not obtain any significant discrepancy with the theoretical evolution of PN nuclei.

However, some objects may effectively deviate from standard evolutionary tracks, as reviewed by Mazzitelli (1992). For example, some PN may have been ejected during helium-shell burning. Others may have nuclei which experienced a helium-shell flash, describing a loop in the HR diagram. Differences in post AGB mass loss efficiencies affect the evolution of PN nuclei in that high mass loss speeds up the evolution: Trams et al (1989) have shown that a mass loss rate of $10^{-7} \mathrm{M}_{\odot} / \mathrm{yr}$ shortens the evolution of a $0.546 \mathrm{M}_{\odot}$ post AGB star by a factor 5 .

\section{GBPN versus other populations of PN}

In the following, we briefly comment on some possible differences and similarities between GBPN and PN in other environments.

\subsection{CHEMICAL COMPOSITION}

The abundances of elements such as $\mathrm{O}, \mathrm{Ne}, \mathrm{S}, \mathrm{Ar}$ indicate the composition of the matter out of which the progenitor stars were formed, thus providing constraints to galactic chemical evolution studies. He, C, and $\mathrm{N}$ have been partly manufactured in the interiors of the stars and then brought to the surface by dredge-up mechanisms occuring during the red giant and $\mathrm{AGB}$ phases. They probe the physical processes occuring during the evolution of intermediate mass stars, and can be used as indicators of the progenitor masses (see Clegg 1989, Ratag et al 1992). For example, the classical subdivision of PN into type I and non type I according to whether the N/O ratio is larger or smaller than 0.5 (Peimbert and TorresPeimbert 1983) is interpreted as a subdivision of the progenitor stars above and below $2 \mathrm{M}_{\odot}$ respectively. This, in turn, leads to an indication of the ages (above and below 3Gyrs on the Maeder and Meynet 1988 scale).

The following table is a compilation of the mean abundances found in the Galactic bulge, in the Galactic disk and in the Magellanic Clouds. The values given for the GBPN are taken from Ratag (1991) and are based on about 120 objects. The ones for the Magellanic Clouds come from Walton et al (1992) and concern over $80 \mathrm{PN}$. The values quoted for the Galactic disk come mainly from Aller and Czyzak (1983) and Aller and Keyes (1987), and concern about 100 objects. 


\section{$\begin{array}{llllll}\operatorname{bgHeH}+12 & \operatorname{bgNH}+12 & \operatorname{bgOHH}+12 & \operatorname{bgNeH}+12 & \operatorname{lgSSH}+12 & \lg \mathrm{AHH}+12\end{array}$

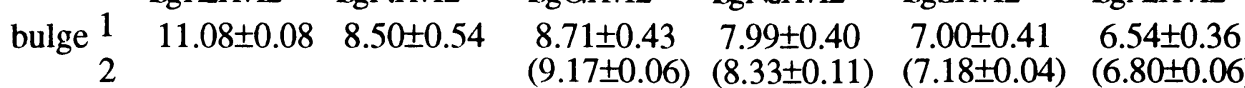 \\ $\begin{array}{llll}(9.17 \pm 0.06) & (8.33 \pm 0.11) & (7.18 \pm 0.04) & (6.80 \pm 0.06)\end{array}$ \\ $\begin{array}{lllllll}\operatorname{disk}^{3} & 11.06 \pm 0.11 & 8.35 \pm 0.45 & 8.64 \pm 0.23 & 8.02 \pm 0.29 & 7.02 \pm 0.35 & 6.52 \pm 0.33\end{array}$ \\ $\begin{array}{lllllll}\text { LMC }^{4} & 11.02 \pm 0.06 & 8.05 \pm 0.71 & 8.40 \pm 0.15 & 7.66 \pm 0.20 & 6.55 \pm 0.18 & 5.91 \pm 0.18 \\ \text { SMC }^{4} & 11.02 \pm 0.08 & 7.58 \pm 0.24 & 8.18 \pm 0.20 & 7.37 \pm 0.23 & 6.42 \pm 0.29 & 5.61+0.22\end{array}$ \\ 1 Ratag et al 1992 \\ 2 extrapolation of Galactic radial gradients towards the bulge, Ratag et al 1992 \\ 3 compilation of disk sample, as quoted in Ratag et al 1992 \\ 4 Walton et al 1992a}

The values in parenthesis are an extrapolation towards the bulge of Galactic gradients derived for PN in the disk. They are on average a factor 3 larger than the abundances effectively measured in the bulge, a fact already noted by Webster (1988). This is not surprising, since kinematically, GBPN are from a different population than disk PN.

The next table gives the average abundances relative to oxygen, in the Galactic bulge and disk and in the Magellanic Clouds.

$\begin{array}{lllll} & \log \mathrm{C} / \mathrm{O} & \log \mathrm{N} / \mathrm{O} & \log \mathrm{Ne} / \mathrm{O} & \log \mathrm{S} / \mathrm{O} \\ \text { bulge } 1,2 & -0.39 \pm 0.41 & -0.16 \pm 0.41 & -0.72 \pm 0.26 & -1.63 \pm 0.31 \\ \text { disk }^{3} & 0.06 \pm 0.21 & -0.30 \pm 0.38 & -0.60 \pm 0.21 & -1.60 \pm 0.32 \\ \text { LMC }^{4} & 0.31 \pm 0.33 & -0.27 \pm 0.33 & -0.73 \pm 0.15 & -1.79 \pm 0.34 \\ \text { SMC }^{4} & 0.53 \pm 0.00 & -0.43 \pm 0.13 & -0.78 \pm 0.20 & -1.72 \pm 0.14\end{array}$

1 Ratag et al 1992

2 Walton et al 1992 b

3 Aller and Keyes 1987 as quoted in Ratag et al 1992 4 Walton et al 1992a

Clearly, type I PN are more frequent in the bulge than in the disk, contrary to what would have been expected for an old population (Ratag et al 1992). Note that, in contrast with Galactic disk PN (Perinotto 1991), large N/O ratios are not preferentially found for objects with a high $\mathrm{O} / \mathrm{H}$. Ratag's conclusion is that, in the bulg, N/O is not an indicator of the progenitors masses, but rather of an earlier $\mathrm{N}$ enrichment of the gas out of which the progenitor stars were formed.

The C/O ratios obtained by Walton et al (1992b) for a dozen of GBPN are significantly smaller than in disk PN, showing that the third dredge up did not operate. This is consistent with the idea that GBPN do not have massive progenitors. 
Comparison of abundances in PN and other stars is a risky task, because the determinations use methods which are not calibrated against each other (see a discussion in Ratag et al 1992 for the bulge and Pagel 1992 for the Magellanic Clouds). But it is meaningful to compare abundances derived for the same types of objects in different environments. For example, Rich (1988) finds strong evidence that Kgiants in the Baade window have higher iron abundances than solar neighbourhood giants.

How is it possible to reconcile the following facts: i) $\mathrm{O} / \mathrm{H}$ ratios similar in the bulge and in the solar neighbourhood ii) ages of the PN populations (as derived from velocity dispersion) of 10-15 Gyrs for the bulge (Pottasch 1984, van der Veen and Habing 1990) and 3-5 Gyrs in the solar neighborhood (Pottasch 1984) iii) N/O and Fe/H ratios larger in the bulge than in the solar neighbourhood? According to Ratag et al (1992), the first two conditions imply that element enhancement must have occured earlier in the bulge than in the disk, the third one indicates a steeper stellar initial mass function in the bulge than in the solar neighbourhood and - possibly - a higher frequency of binary systems.

\subsection{NEBULAR MASSES}

The values of $\mathrm{M}_{\mathrm{i}}$ defined above may be different from the true PN masses, but their largest estimates give an idea of the characteristic total nebular masses. Ratag (1991) finds an upper limit of about $0.6 \mathrm{M}_{\odot}$ for GBPN. In the LMC, the upper limit is also $0.6 \mathrm{M}_{\odot}$ (Barlow 1987, Meatheringham et al 1988). However, it is premature to conclude that PN envelopes have similar masses in the bulge and in the LMC, since Ratag's determinations use angular diameters while those of Barlow and Meatheringham use the densities derived from [O II]. Besides, it is hasardeous to consider upper values in samples of limited size where some natural scatter is expected. Barlow has divided Magellanic Clouds PN into thick or thin, and found that optically thin $P N$ have a mean $\mathrm{M}_{\mathrm{i}}$ of $0.27 \pm 0.06 \mathrm{M}_{\odot}$. A similar study should be made for GBPN. Note that, simulating the sample of GBPN observed with the VLA, Stasinska and Tylenda (1992) obtain satisfactory agreement with observational diagrams for a distribution of total masses of $0.1 \mathrm{M}_{\odot} \pm 0.3 \mathrm{dex}$ (but other solutions may be acceptable).

\subsection{CORE MASSES}

Here again, it is essential to compare values that have been obtained through similar methods based on the same stellar evolutionary tracks.

Present determinations suggest that PN core masses are lower for objects located in the bulge than for PN in the disk: Tylenda et al (1991) find a distribution of central star masses of $0.593 \pm 0.025 \mathrm{M}_{\odot}$ in their bulge 
sample, and $0.615 \pm 0.036 \mathrm{M}_{\odot}$ in their disk sample. However, any conclusion regarding the true distribution of central star masses must fully take into account selection effects.

It seems, though (Ratag 1991), that there is a real lack of GBPN with core masses above $0.6 \mathrm{M}_{\odot}$ in agreement with the upper end of the luminosity distribution of evolved AGB stars in the bulge (Van der Veen and Habing 1990).

\subsection{MASSES OF PROGENITORS}

An insight into the masses of PN progenitors is traditionally provided by the consideration of N/O ratios, as mentionned above. However, we have seen that in the bulge, $\mathrm{N} / \mathrm{O}$ ratios are rather indicative of the early chemical evolution.

Weideman and Koester (1983) have established the existence of a monotonic (though rather flat) relation between the masses of PN nuclei and of their progenitors. In the case of a unique initial-final mass relation, the $\mathrm{O} / \mathrm{H}$ ratio in GBPN should be correlated with the central star mass. Indeed, a PN with a more massive core would have a more massive - thus younger - progenitor, therefore made of chemically enriched material. Ratag (1991) finds no correlation between $\mathrm{O} / \mathrm{H}$ and the core mass, and my own analysis based on the core masses derived in Tylenda et al (1990) supports this conclusion. Probably, variations in mass loss efficiencies on the AGB, significantly alter the initial-final mass relation (Van der Veen 1989, but see also Weideman n1990).

Perhaps a detailed study of the kinematics of GBPN could bring some clues to the progenitor masses.

\subsection{PROPERTIES OF THE GBPN DISCOVERED BY IRAS}

The results discussed sofar concerned mostly PN which were known before the follow up of IRAS sources. An analysis of radio measurements of about 120 GBPN discovered by IRAS is presented in Ratag (1991).

Compared to previously known GBPN, these new GBPN present systematic differences: they have lower radio fluxes and the distribution of infra red exesses is skewed towards much higher values. A first order interpretation would be that IRAS discovered earlier stages of PN, characterized by lower temperature central stars. But Ratag noted that, for the same star temperatures, known GBPN have higher infra red excesses than known disk PN, so that the conditions in the bulge must be different. He retained two possible explanations. One involving the effect of the much stronger (by a factor 50) interstellar radiation in the bulge. The other invoking enhanced $60 \mu$ emission by small iron particles which are likely to be present in the bulge in view of the high Fe abundance. 


\subsection{THE TOTAL NUMBER AND THE FORMATION RATE OF GBPN}

From the $26 \%$ radio detection rate in a subsample of the 1683 IRAS sources with PN colours (Ratag 1991), one expects a total of 450 IRAS detected GBPN. Pottasch (1992) estimates that the total number of GBPN with ages below 8000 years is about 700 .

Usually, the PN birthrate in a given site is obtained by dividing the total number of PN by their mean lifetime. Assuming an expansion velocity of $15 \mathrm{~km} / \mathrm{sec}$, Pottasch (1992) finds a GBPN birthrate of $0.1 \mathrm{PN}$ per year and a specific birthrate of $1.5-310^{-11} \mathrm{PN} / \mathrm{M}_{\odot} / \mathrm{yr}$ assuming a total mass of $1.5-310^{9} \mathrm{M}_{\odot}$ within $3^{\circ}$ of the Galactic center.

However, this number should be considered with care because, for density bounded PN, the time to reach the detection limit depends on the expansion velocity (yet not measured in most GBPN), and for ionization bounded PN, this time is a strong function of the mass of the core and its evolution. Finally, as shown by Isaacman (1979), because of their high velocities, GBPN may be disrupted by ram pressure when crossing a molecular cloud.

Simulations of the GBPN such as initiated by Stasinska et al (1991b and 1992) should provide a better understanding of the PN birthrate and its relation with the evolution of the stellar population in the bulge.

\section{Final remarks}

Many results on the global properties GBPN are still tentative. The firmest ones concern chemical abundances and kinematics.

GBPN studies are developing rapidly. Progress is expected from i) increasing the number of well observed GBPN, ii) including the newly detected GBPN in statistical studies, iii) observing additional quantities like expansion velocitis, molecules... and performing deeper radio sürveys iv) interpreting observational diagrams by taking full account of selection effects.

However, it may be that our quest for understanding the properties of GBPN will never be fulfilled. Indeed, post AGB star evolution depends on many parameters (mass loss efficiencies, chemical composition, binarity...). Will the total number of GBPN be sufficient to perform a statistical analysis taking all this into account?

\section{REFERENCES}

Abell G. O. 1955, PASP 67, 258

Acker A. , Chopinet M., Pottasch S.R., Stenholm B. 1987, A\&AS 71, 163 Acker A., Köppen J., Stenholm B., Raytchev B. 1991, A\&AS 89, 237 
Acker A., Ochsenbein F., Stenholm B., Tylenda R., Marcout J., Schon C., 1992, "Strasbourg-ESO catalogue of galactic planetary nebulae, ESO Acker A., Stenholm B. 1990, A\&AS 86, 219

Aller L.H. 1956, "gaseous nebulae", Chapman \& Hall, London Barlow, M.J., 1987, MNRAS 227, 161

Blöcker T., Schönberner D. 1990 A\&A 240, L11

Clegg R.E.S. 1989, IAU Symp 131, "planetary nebulae", ed. TorrèsPeimbert, Kluwer

Dopita M. A., Henry J. P., Tuohy I.R., Webster B.L., Roberts, E.H., Byun Y.I., Cowie L.L., Songaila A. 1990, APJ 365, 640

Feast M.W. 1968, MNRAS 140, 345

Garcia -Lario P., Manchado A., Pottasch S.R., Suso J., Olling R. 1990, A\&AS 82, 497

Gathier R., Pottasch S.R., Goss W.M., van Gorkom J. 1983 A\&A 128, 325 Gutiérrez-Moreno A., Moreno H., Cortés G. (1991) 383, 174

Habing H.J. 1990, "From Miras to planetary nebulae", ed. M.O.Ménessier \& A.Omont, éditions Frontières

Huggins P.J. 1992, IAU Symp 155, "planetary nebulae", ed Acker, Kluwer Isaacman R. 1981, A\&A 95, 46

Isaacman R. 1983, IAU Symp 103, "planetary nebulae", ed Flower, Reidel Kinman T.D., Feast M.W., Lasker B.M. 1988, AJ 95, 804

Kohoutek, L:, 1992, IAU Symp 155, "planetary nebulae", ed Acker, Kluwer

Köppen J., Acker A., Stenholm B. 1991, A\&A 248, 197

Kudritzki R.P. 1992, IAU Symp 155, "planetary nebulae", ed Acker,

Kluwer

Kudritzki R.P., 1989, IAU Symp 131, "planetary nebulae", ed. TorrèsPeimbert, Kluwer

Manchado A., Pottasch S.R., Garcia-Laro P., Esteban C., Mampaso A. 1989, A\&A 214, 139

Mazzitelli I. 1993, preprint

Meatheringham S.J., Dopita M., Morgan D.H. 1988, APJ 329, 166

Méndez R.H., 1993 A\&A in press

Méndez R.H., Kudritzki R.P., Herrero A. 1993 A\&A 260, 329

Minkowski R., "Galactic structure", ed Blaauw \& Schmidt, unic. of Chicago press

Pagel B.E.J. 1992, "New aspects of Magellanic Cloud research", ed Klare, Springer-Verlag

Peimbert M. 1977, APJ 150, 825

Peimbert M., Torrès-Peimbert S. 1983, IAU Symp 103, "planetary nebulae", ed Flower, Reidel

Perinotto M., 1991, APJS 76, 687

Pottasch S.R. 1984, "Planetary Nebulae", Reidel, 
Pottasch S.R. 1990, A\&A 236, 231

Pottasch S.R. 1992, preprint

Pottasch S.R., Acker A. 1989, A\&A 221, 123

Pottasch S.R., Bignell C., Olling R., Zijlstra A.A. 1988, A\&A 205, 248

Pottasch S.R., Ratag M.A., Olling R.,1990, "From Miras to planetary

nebulae", ed. M.O.Ménessier \& A.Omont, éditions Frontières

Pottasch S.R., Zijlstra A.A. 1992, A\&A 256, 251

Ratag M.A. 1991, thesis, University of Groningen

Ratag M.A., Pottasch S. R. 1991, A\&AS 91, 481

Ratag M.A., Pottasch S.R., Dennefeld M., Menzies J. 1992, A\&A 255, 255

Ratag M.A., Pottasch S.R., Zijlstra A.A., Menzies J. 1990, A\&A 233, 181

Rich R.M. 1988, AJ 95, 828

Schönberner D. 1979 A\&A 79, 108,

Schönberner D. 1983, APJ 272, 708

Schönberner D., 1989, IAU Symp 131, "planetary nebulae", ed. TorrèsPeimbert, Kluwer

Schönberner D., Tylenda R., 1990, A\&A 234, 439

Stasinska G., Fresneau A., Gameiro J..F., Acker A. 1991b, A\&A 252, 762

Stasinska G., Tylenda R., 1992, IAU Symp 155, "planetary nebulae", ed Acker, Kluwer

Stasinska G., Tylenda, R., Acker A., Stenholm B. 1991a, A\&A 247, 173

Trams N.R., Waters L.B.F.M., Waelkens H.J.G.L.M., van der Veen W.E.C.J., 1989, A\&A 218, L1

Tylenda R., 1992, IAU Symp 155, "planetary nebulae", ed Acker, Kluwer

Tylenda, R., Stasinska G., Acker A., Stenholm B. 1991, A\&A 246, 221

van der Veen W.E.C.J., 1989, A\&A 210, 127

van der Veen W.E.C.J., Habing H.J. 1990, A\&A

Vassiliadis E., Wood P.R., 1992, in preparation

Walton, N.A., Barlow M.J., Clegg R.E.S. 1992b, IAU Symp 155,

"planetary nebulae", ed Acker, Kluwer

Walton, N.A., Barlow M.J., Monk D.J., Clegg R.E.S. 1992a, "New

aspects of Magellanic Cloud research", ed Klare, Springer-Verlag

Webster B.L. 1975, MNRAS 173, 437

Webster B.L. 1976, 174, 513

Webster B.L. 1988, 230, 377

Weidemann V., 1990, A R A\&A 28, 103

Weidemann V., Koester D. 1983, A\&A121, 77

Zijlstra A.A. 1990 A\&A 234, 387

Zijlstra A.A., Pottasch S.R. 1989, A\&A 216, 245 


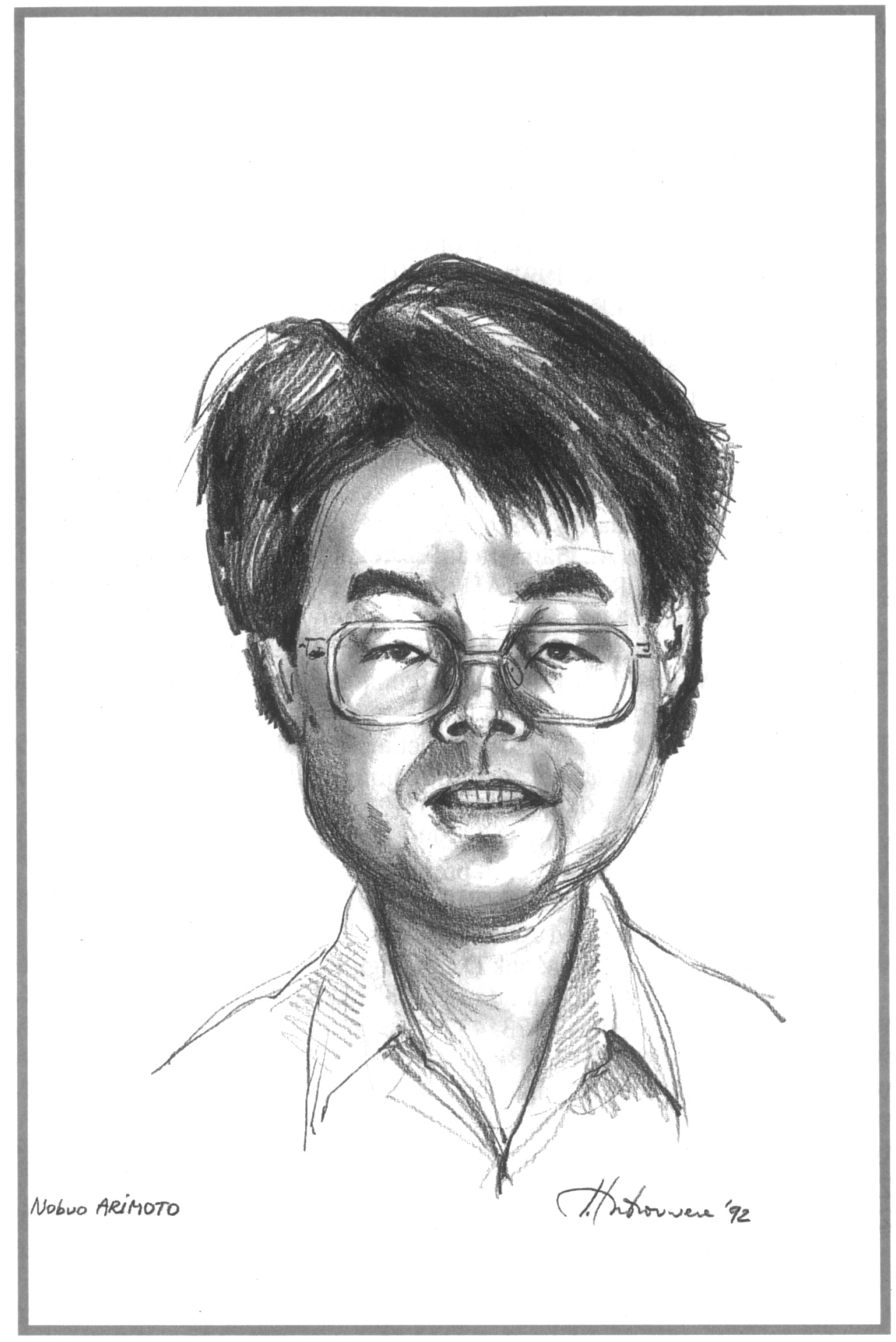

